\title{
Astrobiology on Mars: Organic Chemical Evolution on an Earth-like Planet
}

POC: Alfonso Davila

NASA Ames Research Center

Ph: 650-604-0695

Email: alfonso.davila@nasa.gov

\section{Contributors in alphabetical order:}

Morgan Cable (JPL); David Catling (U. Washington); James Cleaves (Tokyo Inst. Tech.); Jennifer Eigenbrode (NASA); Devan Nisson (Princeton U.); Michel Nuevo (BAER Institute); Sarah Johnson (Georgetown U.); Andrew Steele (Carnegie Inst. of Washington); Jennifer Stern (NASA).

Signatories in alphabetical order: Jennifer Blank (Blue Marble Space Inst.); David Flannery (Queensland U. of Tech.); James Lewis (NASA); Maeva Millan (NASA); Tullis Onstott (Princeton U.); Charity Phillips-Lander (SWRI); Svetlana Shkolyar (USRA/NASA) 
This white paper recommends that the next decade of Mars exploration prioritizes research into the chemistry, distribution, and origin of organic matter in martian geologic materials, both in situ and in samples returned to Earth

\section{Introduction}

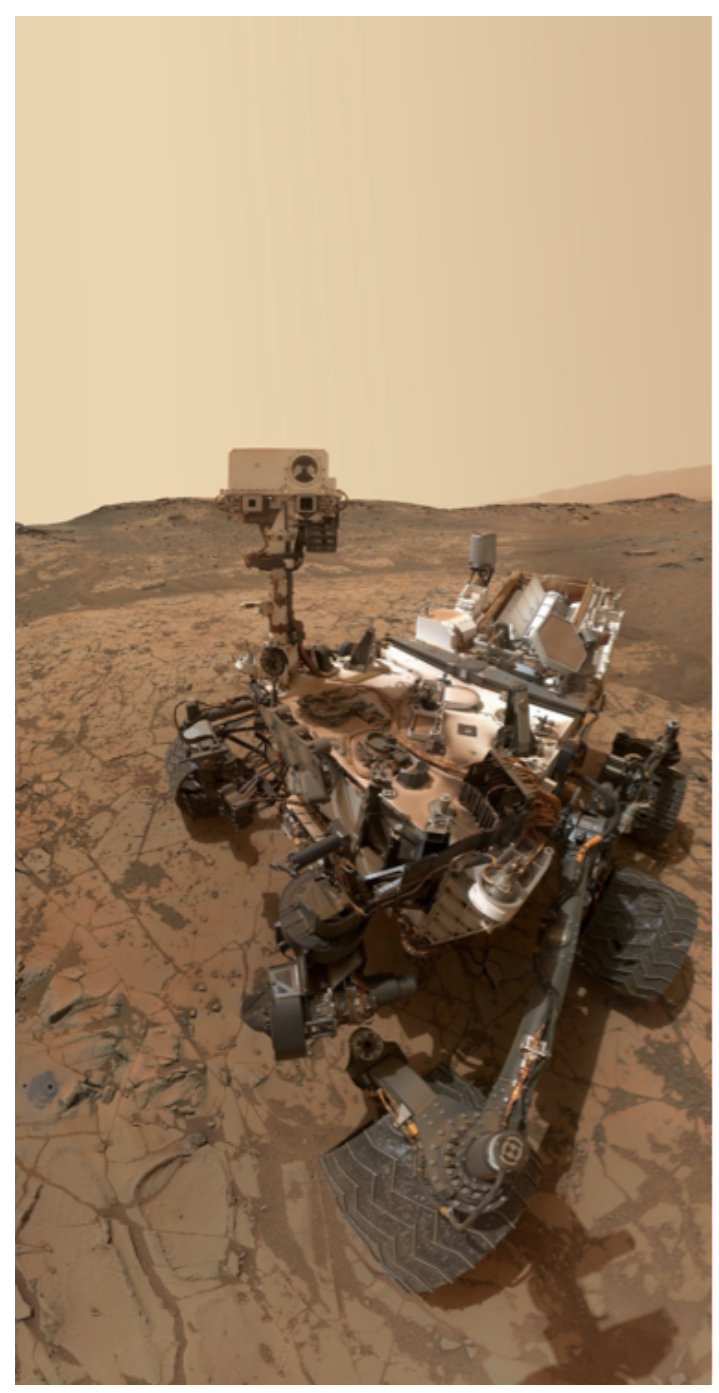

Life, when considered in a planetary context, is one end member in the continuum of organic chemical evolution (OCE). The origin of life is likely the result of a series of abiotic chemical reactions which yielded increasingly complex organic molecules and molecular assemblages from simpler ones, leading to autocatalytic cycles and eventually to the emergence of the first self-replicating organism. From that point onwards, biochemistry drove OCE via chemical interactions between many informationally ordered organic macromolecules.

The discovery of organic matter in several martian meteorites and in lake sediments at Gale Crater were stunning successes of the last decade of Mars exploration. Martian organic matter (MOM) has been detected in the form of a macromolecular carbon (MMC) phase that includes polycyclic aromatic hydrocarbons (PAHs), as well as nitrogen-, oxygen-, and sulfur-bearing species. Pyrolysis products of Gale Crater lake sediments released between $500-820^{\circ} \mathrm{C}$ revealed a highly refractory organic $\mathrm{MMC}$ component. More volatile organic molecules might also be present in the sediments, based on the identification of chlorobenzene and dichloroalkanes by gas chromatography coupled to mass spectrometry. However, chemical analyses of this lower volatility component of martian organics have been challenging due to the chemical reactivity of martian soil as well as instrument background sources.

The presence of organic matter in martian geological materials suggests that OCE was an active process on Mars in the past, that could still be ongoing now. This organic material could hold the clues to determine whether the transition from geochemistry to biochemistry may have occurred twice in the Solar System, and led to an independent origin of life on Mars. 


\section{BIG QUESTIONS}

\section{Does martian organic matter contain biological building blocks?}

The discovery of biological building blocks in martian samples could revolutionize our understanding of the origin of life

Every martian organic molecule is an important bit of chemical information. But the discovery of certain classes of organic compounds in martian geologic materials would truly increase our understanding of OCE on Earth-like planets. These classes include amino acids, fatty acids, nucleobases, and sugars. These classes of compounds fulfill two requirements: (i) they are readily synthesized under prebiotic conditions, as evidenced by their detection in carbonaceous meteorites; and (ii) they are biological building blocks, playing an essential role in contemporary terrestrial biochemistry.

The discovery of amino acids, fatty acids, nucleobases, or sugars in martian geologic materials could significantly advance existing models of OCE. For decades, research into this topic has relied on two approaches: laboratory abiotic synthesis of organic molecules under plausible environmental conditions, and analyses of primordial extraterrestrial organics collected on Earth or returned from space missions (Stardust, Hayabusa, Hayabusa2, OSIRIS-REx). Both approaches have shed light on possible sources of prebiotic building blocks (endogenous and exogenous) and the chemical pathways that could lead from small organic molecules to macromolecules, and eventually to biomolecules and life (noting that none of these pathways has been actually confirmed in a planetary environment). However, questions still remain as to the relative contribution of endogenous and exogenous sources in planetary settings that are considerably more complex and dynamic than typically studied in laboratory simulations. The environmental conditions that favor the accumulation or destruction of prebiotic building blocks remain a critical knowledge gap. Of interest would be to investigate how endogenous and exogenous mixtures of prebiotic building blocks evolve in habitable environments in the absence of life, as this could provide new clues regarding the steps that lead from prebiotic chemistry to biochemistry in our own planet.

Organic matter in martian geologic materials could represent a third and new approach to study OCE under planetary conditions, and may provide some context to the conditions that shaped prebiotic chemistry on early Earth, on other planets in our Solar System, and in other Solar System bodies (icy moons, comets, asteroids). The presence of prebiotic building blocks in martian organic matter would be an opportunity to test current models for the evolution of primordial biological traits, such as the development in aqueous solutions of amphiphilic membranes derived from either exogenous or endogenous organic materials, or the spontaneous formation of short polymers of amino acids that could be early precursors of catalytic biopolymers. 


\section{What are the sources of martian organic matter?}

Martian organic matter could hold the key to determine whether Mars ever supported, or still supports, life

The transition from geochemistry to biochemistry may have happened only once in the history of the Universe, namely, on Earth, or it may occur frequently wherever conditions are favorable. We do not know exactly when, where, or how this transition occurred on Earth, as the geologic record of that period has largely been lost because of plate tectonics, erosion, and catastrophic events. Analyses of MOM could address this question directly by determining whether these materials (i) were produced by abiotic reactions on Mars, (ii) were delivered to Mars after being produced by abiotic reactions outside of Mars, or (iii) are the products of extinct or extant life on Mars, if sufficiently stringent diagnostic criteria can be developed.

Biotic sources of organic matter leave diagnostic fingerprints (biosignatures) in the composition and structure of the organic molecules they generate. Some of these fingerprints can still be detected even after billions of years of thermal, radiation-induced, and/or chemical alteration. For example, the oldest evidence of life on Earth ( $\geq 3.8$ billion years ago) is recorded in the ${ }^{12} \mathrm{C} /{ }^{13} \mathrm{C}$ isotopic values of carbonaceous matter in sedimentary rocks. Analyses of the Yellowknife Bay mudstone formation in Gale Crater indicate the existence on Mars of a fluvio-lacustrine environment capable of supporting life as we know it $\sim 3.5 \mathrm{Gyr}$ ago. Isotopic analyses of organic matter in the mudstone sediments are consistent with exogenous sources (meteorites; interplanetary dust) and endogenous sources (photochemistry; electrochemical reduction of $\mathrm{CO}_{2}$ ).

Exogenous delivery of organic matter has likely been continuous throughout Mars' history, as it has been and continues to be on Earth, albeit at diminishing rates since planetary accretion. Estimates of the flux and organic content of meteoritic materials and the destruction rates of organic matter by UV radiation, suggest that there could be a steady state residual of several parts per million of meteoritic organics in the martian regolith. These values overlap with estimated organic abundances in sediments at Gale Crater, and might represent a global baseline for exogenous organic material near the martian surface. Other endogenous abiotic pathways of organic synthesis and OCE could have operated synchronously or sequentially throughout Mars' history (Fig 1). Exogenous and endogenous abiotic sources would have generated feedstocks of organic molecules analogous to those that were created on the prebiotic Earth. This trove of abiotic organic chemical information would be difficult to reproduce in laboratory settings, and it is unlikely to be found in the other inner planets, including on Earth, where biogenic organic matter is pervasive on the surface and in the subsurface.

Mars' subsurface is also of great interest for determining whether life exists now or existed in the past. Water-bearing subsurface aquifers, stabilized by internal heat generation, might have sustained a protracted history of OCE, perhaps up to recent times. Low molecular weight organics could be produced on Earth abiotically through water-rock reactions including serpentinization, Fischer-Tropsch type synthesis. Organic salts, such as oxalates, acetates, and benzenecarboxylates, could be generated from more complex organic matter by oxidation and radiation. Mars represents an ideal natural laboratory to profile the transition from an irradiated and oxidized surface organic record to less-altered organic matter at depth. Subsurface organic matter and inorganic energy sources, such as $\mathrm{H}_{2}$ produced radiolytically and/or via serpentinization of ultramafic host rock, could be the basis for a deep subsurface biosphere of chemolithotrophic microorganisms analogous to that found in Earth's crust. 


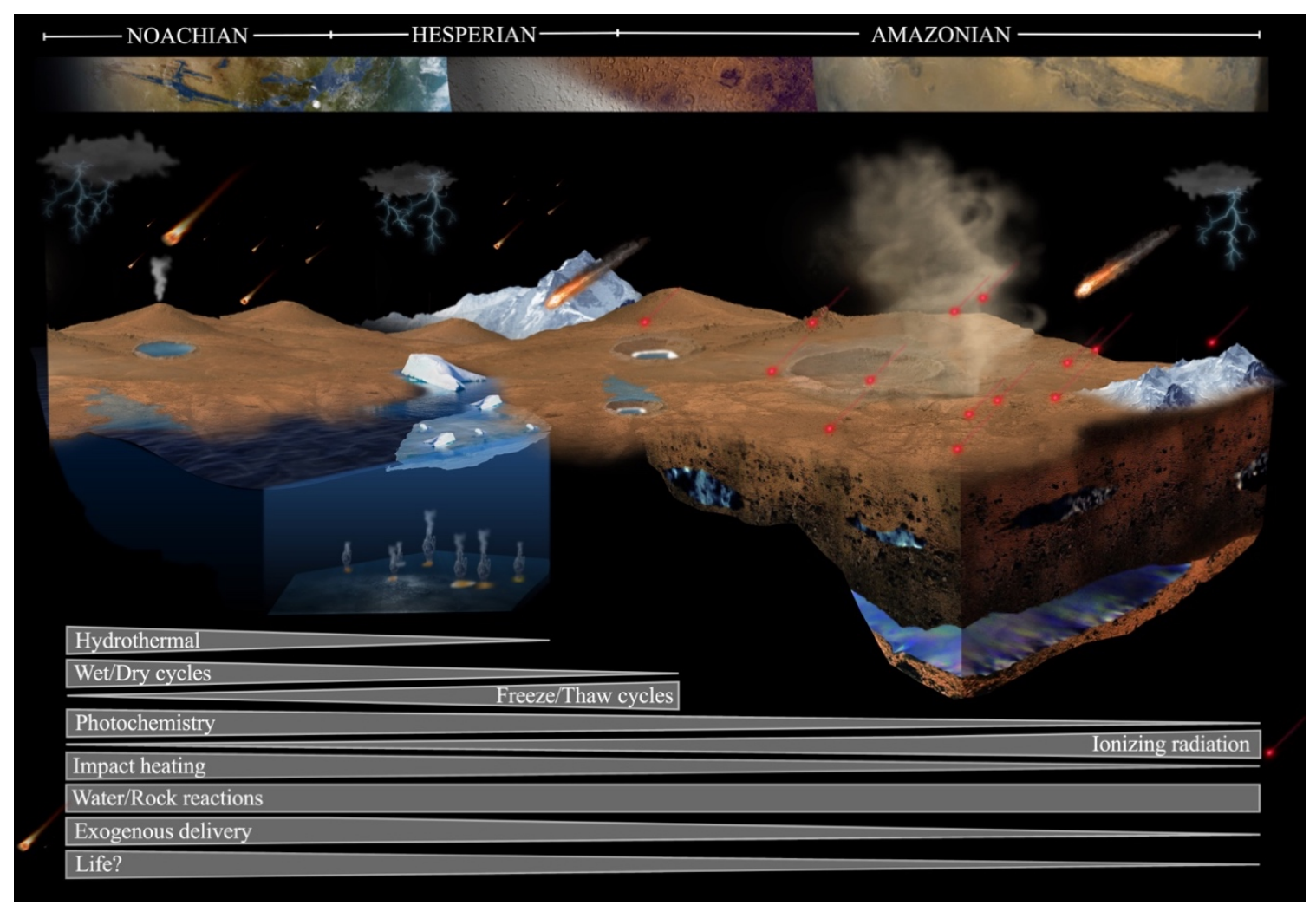

Figure 1. Endogenous and exogenous sources of organic matter throughout Mars' history.

\section{What does OCE on Mars tell us about the evolution of the Solar System?}

\section{Studies of martian organic matter will help shift perspectives of OCE from Earth-centric} to Solar System-wide

Studies of organic chemistry on Earth, Mars, and in some ocean worlds in the outer Solar System (icy satellites of giant planets that probably possess a global water ocean underneath their surface ice layers) could provide important synergies, primarily because all utilize the same solvent: water (Titan's hydrocarbon lakes are excluded from this assessment, though Titan may contain a deep water ocean as well). Any organic chemistry that occurs in water will be constrained by the physical and chemical properties of the aqueous solution (i.e., presence of salts, solubility, reaction rates, catalytic activity, etc.). Furthermore, the 'building block' molecules that define organic chemistry - biotic or abiotic — should be common across Solar System environments, as evidenced by the widespread occurrence of amino acids, lipids and other organics that are deposited via cometary and meteoritic impacts.

At the same time, studies of organic chemistry on Earth, Mars, and in ocean worlds could provide the means to test existing, and sometimes conflicting, hypotheses regarding OCE. Ocean worlds constitute a unique case with their relatively stable aqueous environments that have been unaffected by wet/dry cycles and radiation for long periods of time. In contrast, Mars provides a tale of two planets. For the first billion years, Mars was likely geochemically as diverse as the early Earth, harboring a broad range of temperatures, pressures, $\mathrm{pH}$, water activity, and redox 
conditions. But for the last three billion years, Mars has been a desert planet, where hydrological processes at the surface have been largely dominated by wet/dry cycles under low-pressure regimes in the pore space of crustal materials (sediments and regolith).

\section{SCIENCE INVESTIGATIONS}

\section{Studies of OCE on Mars align directly with Goal 1 of MEPAG's Science Goals, Objectives, Investigations, and Priorities}

1. Constrain crustal inventories of organic molecules over time. Investigations in this objective are primarily focused on establishing whether organic compounds exist in martian surface or subsurface environments, particularly in environments harboring liquid water activity. (Addresses MEPAG Goal 1, Sub-Objectives A1 and B1).

Priority measurements: monomer ${ }^{1}$ abundances, enantiomeric ${ }^{2}$ excesses, structure and composition of organic molecules, including polymers, molecular-size distributions and stable isotopic abundances in possible organic metabolic reactants or products. Also of particular importance are concomitant measurements of exposure ages of surface materials from either in situ geochronology or relative dating methods.

2. Assess the preservation potential of organic matter near the surface and as a function of depth. Investigations in this objective are focused on establishing the potential of a given environment to preserve organic matter between the time of deposition/formation and the time of detection. (Addresses MEPAG Goal 1, Sub-Objective A3).

Priority measurements: redox changes and rates in surface and subsurface environments (including the determination of the effects of regolith and rock burial on the shielding from ionizing radiation); investigation of potential processes that influence isotopic or stereochemical $^{3}$ information, and microscopic studies of rock samples. Laboratory research and studies of Mars analog environments can provide important insights pertinent to preserving organic matter in martian geological materials.

3. Constrain the atmosphere, surface, and subsurface processes through which organic molecules could have formed and evolved throughout martian history. Investigations in this objective are focused on the identification of potential mechanisms responsible for organic synthesis and evaluation of their presence in the martian atmosphere and crust, such as radiation from energetic photons and/or particles, impact shock heating, mineral catalysis, hydrothermalism, or serpentinization. (Addresses MEPAG Goal 1, Sub-Objective B2).

Priority measurements: effects of inherent and photolytic radiation on organics, and mineral-organic co-occurrence and relationships. These relational measurements aim at deciding which mechanisms contribute to OCE in a given environment.

\footnotetext{
${ }^{1}$ Monomers are molecules that can bond covalently to form a repeating polymer such as amino acids, sugars, and nucleotides reacting to form peptides, polysaccharides, and nucleic acids, respectively.

2 Enantiomers are chiral molecules that are mirror images of each other, such as L/D-amino acids. Enantiomeric excess refers to the dominance of one enantiomer over the other one. Biology typically uses only one enantiomer of a given chiral compound, i.e., only L-amino acids for proteins, only D-sugars for the backbones of DNA and RNA.

${ }^{3}$ Stereochemistry refers to the spatial arrangement of atoms in chiral molecules.
} 


\section{MISSION CONCEPTS AND ARCHITECTURES}

In situ studies of OCE on Mars will require access to the surface and subsurface with landers or rovers, and a level of resources equivalent to New Frontiers-class missions

The return to Earth of a carefully selected set of geologic samples from Jezero Crater will represent a significant opportunity to advance our understanding of the organic content in materials on Mars' surface. However, surface sedimentary deposits in Jezero Crater only provide a record of a tiny slice of Mars's geological history. Attributes that make Jezero Crater a compelling site for exploration exist in other regions where OCE, habitable conditions, and potentially life, might have occurred at many stages of Mars' history. These include hydrothermal deposits, salt-rich evaporitic deposits, icy regoliths, diverse sedimentary deposits, and the deep subsurface (Fig 2). Further efforts to explore a broader parameter space of environments in situ, particularly those that could have been at some time habitable, must be considered.

In situ investigations of organic chemistry and $\mathrm{OCE}$ will require access to the martian surface and subsurface. Many of the scientifically interesting sites are located in Mars' southern highlands, which demand more complex landing operations than has been achieved to date. Some of these locations are confined to relatively small areas and demand high landing accuracy, perhaps aided by intelligent and autonomous navigation systems. The need for surface mobility ought to be considered on a mission-specific basis. High landing accuracy could mitigate the need for long-range mobility. A minimal amount of mobility might still be required once on the surface to access the most interesting sampling locations, or to provide access to a sufficiently large diversity of samples to support meaningful data interpretation. Surface robotic missions to date have only sampled to depths of a few centimeters, rendering the martian subsurface largely unexplored. The subsurface should be considered an exciting new frontier for Mars exploration, and a particularly promising target environment to investigate OCE.

The Discovery-class Insight mission successfully landed on Mars on a cost-capped budget of $\$ 500$ $\$ 600 \mathrm{M}$. Insight was a static platform and landed at

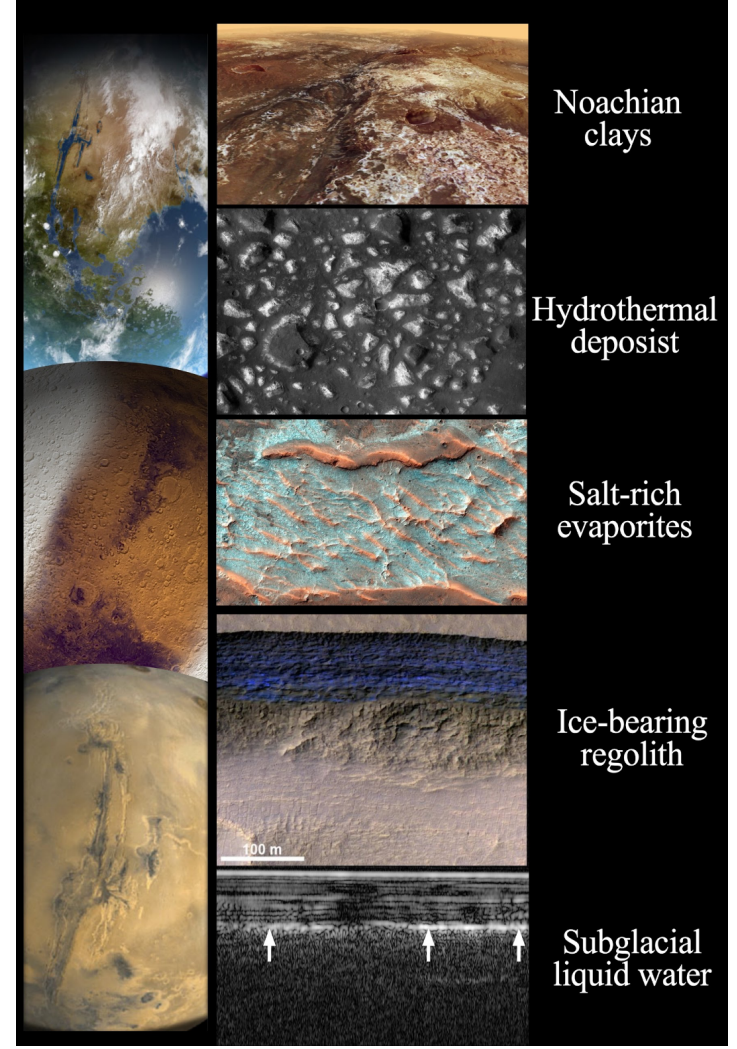

Figure 2. Many diverse habitable environments that could have supported OCE might have existed throughout the history of Mars, from the warmer and wetter Noachian period (top) to the hypothermal and hyperarid Amazonian period (bottom). Elysium Planitia (elevation: $-2,600 \mathrm{~m}$ ). The technological requirements to land in more confined regions located at higher elevations, and with a short-to-long range degree of mobility, as well as the technology needed to access the subsurface, will require more resources in line with a New Frontiers-class mission with a \$800M-\$1B budget. 


\section{NEW TECHNOLOGIES}

The next phase of organic chemistry investigations on Mars will move from 'detection' to 'characterization'. New technologies that focus on sample preparation and compoundspecific isotope analysis ought to be prioritized for the next decade.

It has taken multiple attempts to detect MOM in situ on Mars to produce our present state of knowledge. Much has been learned in the process regarding the factors that limit the preservation of organic matter near the surface; chiefly amongst them are destruction by radiation and oxidation. Important lessons have been learned regarding factors that interfere with organic chemical analyses, such as the presence of perchlorates that cause the combustion of organic compounds at temperatures below $400^{\circ} \mathrm{C}$, which makes the detection of even simple compounds such as amino acids challenging with established flight instruments. Lessons learned from past efforts will undoubtedly have to be applied in the next phase of organic chemistry investigations, and new technological capabilities will be required to move from 'detection' to 'characterization'.

The characterization of organic compounds in a laboratory setting typically requires sample preparation steps. The highest sensitivity in organic molecule detection and characterization typically requires the separation of the organic fraction from the inorganic matrix. Thermal volatilization has been implemented in several Martian missions to separate volatile organic compounds prior to mass spectrometry analyses, but it has difficult due to matrix interference effects. An alternative approach is solvent extraction, which has several advantages such as avoiding thermal damage. NASA's 2015 Technological Roadmap acknowledged that "In the ongoing search for life, wet chemical analysis approaches and sensors need to be developed to allow biological signatures or organic material to be characterized". Sample preparation systems for in situ organic chemical analyses remain a technological goal for Mars exploration.

Compound-specific isotope analysis (CSIA) has been useful in deciphering complex organic mixtures in planetary materials, and also for teasing out biological effects on the chemical composition of certain types of organic compounds, which tend to fractionate stable isotopes of bioessential elements (e.g., enrichment of ${ }^{12} \mathrm{C}$ over ${ }^{13} \mathrm{C}$ ). CSIA can provide insights into the sources and reaction mechanisms that contribute to organic assemblages in martian samples. The development of flight technologies such as CSIA, which enable measurements of isotopic ratios of $\mathrm{C}, \mathrm{H}, \mathrm{N}$, $O, S$, and other bio-essential elements ought to be prioritized for the next decade.

\section{SUMMARY}

- The next decade of Mars exploration ought to prioritize research into the chemistry, distribution, and origin of organic matter in geologic materials, both in situ and in samples returned to Earth.

- Organic matter could hold the key to determine if Mars ever supported, or still supports, life.

- The discovery of biological building blocks in martian organic matter could revolutionize our understanding of the origin of life, and would help shift perspectives of OCE from Earth-centric to Solar System-wide.

- In situ studies of organic matter on Mars will require access to the surface and subsurface with landers or rovers, and a level of resources equivalent to New Frontiers-class missions.

- The next phase of organic chemistry investigations on Mars will move from 'detection' to 'characterization'. New technologies that focus on sample preparation and compound-specific isotope analysis ought to be prioritized for the next decade. 